\title{
Comparison of different scoring systems for immunohistochemical staining
}

\author{
E J Adams, J A Green, A H Clark, J H Youngson
}

\begin{abstract}
Aim-To carry out an objective assessment of two systems of scoring immunohistochemical staining, evaluating interobserver and intraobserver error.

Methods-92 cervical tumours underwent immunohistochemical staining for p53 and epidermal growth factor receptor. Staining was assessed using two methods: a standard 4 point scale and a descriptive method, performed by three observers. Interobserver and intraobserver error were assessed for both scoring methods. Results-In terms of interobserver error between three observers, no difference was found between a simple 4 point scale method of evaluation and the use of a highly circumscribed method. In all evaluations, interobserver error was scored as moderate ( $\mathrm{kw} 0.48-0.49$ ). However, evaluation of immunohistochemical staining by a panel of observers led to a marked improvement in the interobserver error scores ( $\mathrm{kw}$ 0.63).

Conclusions-There should be standardisation of immunohistochemical staining and scoring methods. More attention should be paid to measurement of interobserver and intraobserver error in studies. Use of a panel of tissue control slides and consensus scoring by several observers can lead to improvement in reproducibility.

(F Clin Pathol 1999;52:75-77)
\end{abstract}

Department of

Medicine, University

of Liverpool,

Liverpool, UK

E J Adams

J A Green

Pathology

Department, Arrowe

Park Hospital, Wirral,

UK

A H Clark

Merseyside and Cheshire Cancer Registry, North Cheshire Health, Muspratt Building, University of Liverpool, UK

$\mathrm{J}$ H Youngson

Correspondence to: Dr E J Adams, Department of Urodynamics, Liverpool

Women's Hospital, Liverpool L8 7SS, UK.

Accepted for publication 18 September 1998
Keywords: immunohistochemistry; scoring system; interobserver error

Immunohistochemical staining techniques are an important research tool, and an increasing array of antibodies is being produced which can identify a range of tissue antigens, tumour markers, and oncogene products in both frozen and archival material. However, these techniques share a colour reaction as the end point of the experiment, which is both subjective and difficult to quantitate, thus limiting the power of the technique. Most researchers use a form of 3 or 4 point scale to define intensity and distribution of staining. Measurement of interobserver error is rarely carried out and consequently comparison of results from different centres is difficult to achieve. The task force of the EORTC-GCCG has recently reported on a protocol for immunohistochemical staining but actual scoring systems were not specifically addressed. ${ }^{1}$ We report an objective comparison between a widely used 4 point scale and a scoring system which was originally proposed by Fisher for the detection of nuclear p 53 protein in tissue sections, based on highly circumscribed definitions. ${ }^{2}$ We adapted the Fisher scoring system for use with the cell membrane localised epidermal growth factor (EGF) receptor.

\section{Methods}

Following identification of cases by the regional cancer registry, a series of 277 paraffin embedded cervical carcinomas was obtained from Arrowe Park Hospital, Wirral, which had been diagnosed in Wirral Health District between 1981 and 1992. Thirty samples of normal cervix were also obtained to act as controls, and these were processed alongside the tumour samples. The original H\&E slides for each tumour were reviewed by a single consultant pathologist, following which representative paraffin embedded blocks of each case were selected to provide the maximum amount of tumour for analysis. Between 1981 and 1992, all tissues received at Arrowe Park Hospital histopathology department had undergone fixation in $10 \%$ buffered formolsaline for 24 hours before paraffin embedding. Of the 277 tumours in the study, scoring data for both scoring systems were available for the first 92 consecutive cases, and our analysis is based on these tumours.

Sections from each block of $5 \mu \mathrm{m}$ thickness were cut and floated on a water bath at $55^{\circ} \mathrm{C}$ before being placed on to plain glass slides and dried at $37^{\circ} \mathrm{C}$ overnight. Haematoxylin and eosin $(\mathrm{H} \& \mathrm{E})$ stains were repeated and mucin stains were carried out using the PAS/diastase method to confirm the histological diagnosis in each case, according to the criteria of Buckley and Fox. ${ }^{3}$ Immunohistochemical staining was carried out on all tumour samples to identify p53 and epidermal growth factor receptor (EGFr) protein expression.

The avidin-biotin-peroxidase method of immunohistochemistry ${ }^{4}$ was used for both proteins. Following dewaxing and dehydration through alcohols, endogenous peroxidase activity was blocked with 3\% hydrogen peroxide. The antibody used to stain p53 protein was a rabbit polyclonal antibody, CM-1 (Novocastra), which reacts with both mutant and wild-type p53, and the positive control tissue used was a preparation of paraffin embedded monkey Cos cells, transfected with a plasmid containing the human p53 gene (provided by Dr J Jenkins, Marie Curie Institute, Oxted, UK). The CM-1 antibody was optimised at a concentration of 1:600, diluted in phosphate buffered saline (PBS) with bovine serum albumin (BSA), and then incubated with the tumour sections for 30 minutes at room 
Table 1 p53 and EGFr immunocytochemical scoring system (adapted from Fisher et al, $\left.1994^{2}\right)$

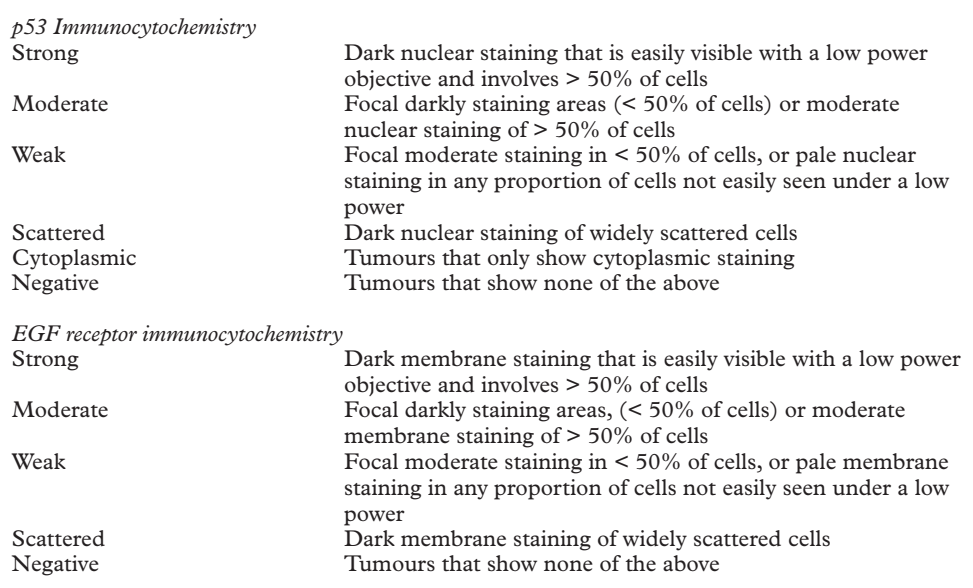

temperature. E30, a mouse monoclonal antibody to the protein portion of the extracellular domain of the EGF receptor (Biogenix), was used to identify EGF receptor expression, and the positive control tissue for EGFr was normal human placental membrane. Before adding E30 antibody, pepsin digestion was necessary, using a solution of $0.1 \%$ pepsin in $0.01 \mathrm{~N} \mathrm{HCl}$ for 10 minutes at $37^{\circ} \mathrm{C}$. E30 antibody was used as supplied by the manufacturers in a 1:20 dilution, and required a two hour incubation at $37^{\circ} \mathrm{C}$. Negative controls were included in each staining run-for CM-1, normal rabbit serum was applied to positive control slides; E30 antibody is supplied as a kit containing an appropriate negative control serum, derived from mouse ascites. A further negative control was PBS containing $0.1 \% \mathrm{BSA}$ on positive control sections.

The resulting slides were reviewed independently by three experienced observers. Intensity was assessed by comparison with the positive control sections and by review of a

Table 2 Weighted $\kappa$ for 4 point scoring system compared with Fisher scoring system

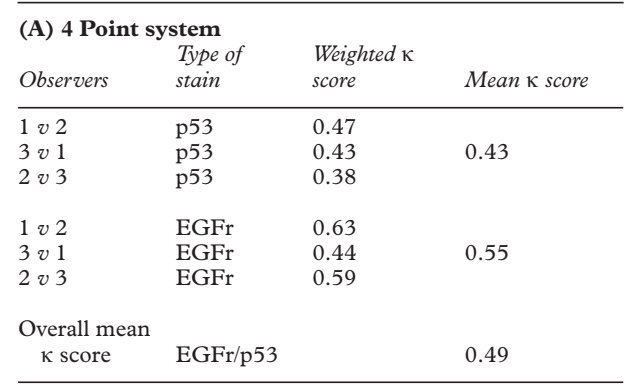

(B) Fisher scoring system

\begin{tabular}{|c|c|c|c|c|c|}
\hline \multirow[b]{2}{*}{ Observers } & \multirow[b]{2}{*}{$\begin{array}{l}\text { Type of } \\
\text { stain }\end{array}$} & \multicolumn{2}{|c|}{ Before discussion } & \multicolumn{2}{|c|}{ After discussion } \\
\hline & & $\begin{array}{l}\text { Weighted } \kappa \\
\text { score }\end{array}$ & Mean $\kappa$ score & $\begin{array}{l}\text { Weighted } \kappa \\
\text { score }\end{array}$ & Mean $\kappa$ score \\
\hline $1 v 2$ & p53 & 0.46 & & 0.54 & \\
\hline $3 v 1$ & p53 & 0.45 & 0.49 & 0.61 & 0.61 \\
\hline $2 v 3$ & p53 & 0.57 & & 0.69 & \\
\hline $1 v 2$ & EGFr & 0.37 & & 0.62 & \\
\hline $3 v 1$ & EGFr & 0.49 & 0.47 & 0.60 & 0.65 \\
\hline $2 v 3$ & EGFr & 0.51 & & 0.73 & \\
\hline $\begin{array}{l}\text { Overall mean } \\
\kappa \text { score }\end{array}$ & $\mathrm{EGFr} / \mathrm{p} 53$ & & 0.48 & & 0.63 \\
\hline
\end{tabular}

series of tissue controls, which were tumour slides which had been selected by the three observers as examples of each of the scores.

The intensity and distribution of positive staining was evaluated using two scoring systems, performed six months apart by the three observers. The first evaluation employed a standard 4 point scale for intensity, with slides being scored as negative,,+++ , or +++ , with the positive control slide being taken as +++ . The second evaluation employed a more circumscribed scoring system, originally proposed by Fisher, ${ }^{2}$ with a modified score being used for EGFr staining to take account of the localisation of EGFr to the cell membrane rather than the nucleus or cytoplasm. As shown in table 1 , this scoring system combines both the intensity and distribution of positive staining as a one word score.

Following the independent evaluation of the immunohistochemical staining, there was wide variation between the observers in scoring 16 tumours. These slides were discussed at a separate evaluation in order to reach a consensus on the correct score. In the subsequent analysis, the interobserver error was calculated using both the original and the consensus scores.

All scores provided by the three observers were entered into a database using Lotus 1-2-3 Approach software, and were analysed using the weighted $\kappa$ statistic ( $\mathrm{Kw}$ ) for interobserver error. ${ }^{5}$ Intraobserver error was also assessed.

\section{Results}

Strong p53 staining was identified in $7 \%$ of the tumours, moderate staining in $21 \%$, scattered staining in $2 \%$, weak staining in $40 \%$, and negative staining in $27 \%$. Pure cytoplasmic p53 staining was found in $4 \%$ of the tumours. All samples of normal cervix were negative for p53. Strong EGFr staining occurred in $10 \%$ of the tumours, moderate staining in $25 \%$, weak staining in $43 \%$, and negative staining in $23 \%$. There were no scores of "scattered" for this antibody. The majority of normal cervices showed negative staining, with 39\% having weak or moderate EGFr positivity, which was confined to the epithelium of the cervix. Since the EGF receptor is found at low levels on the surface of all normal cell types (except nerve tissue), a degree of positive staining was expected. This has been described previously. ${ }^{6}$

The interobserver agreement between the three observers was calculated for each of the two scoring systems and both antibodies for the first 92 tumours. The degree of agreement between each of the observers, both before and after panel discussion, and the mean weighted $\kappa$ values, are shown in table 2 . The mean weighted $\kappa$ value for intraobserver variation was 0.32 (range 0.24 to 0.47 ).

\section{Discussion}

The level of p53 positivity identified is slightly lower than that reported by some investigators, but differences in scoring methods often make comparisons between studies difficult. For example Holm et al reported $62 \%$ of 238 invasive cervical tumours to be positive for p53 
expression, using the antibodies CM-1 and $\mathrm{PAb} 1801 .^{7}$ However, their study included many tumours in which only an occasional nucleus was positive and only $9 \%$ of tumours in the study showed strong staining involving more than $5 \%$ of the tumour cells. Oka et al examined 192 stage III squamous carcinomas of the cervix using CM-1 antibody and employed a graded scoring system similar to the one used in our study. ${ }^{8}$ They identified $7 \%$ of the tumours as having strongly positive staining, while $51 \%$ were negative.

The level of EGFr overexpression found in this study is similar to that described by Kohler et $a l,{ }^{9}$ who found that $40 \%$ (16 of 42 cervical tumours) were positive by immunocytochemistry and EGF binding assay.

The relevance of $\kappa$ statistic values has been described by Landis and Koch. ${ }^{10}$ Values of less than 0.2 are described as "poor," those between 0.2 and 0.4 are "fair," and values between 0.4 and 0.6 indicate moderate agreement; values of 0.6 to 0.8 show good agreement, while those between 0.8 and 1.0 show excellent agreement. In practice, any value above 0.5 indicates reasonable agreement between the observers.

No difference was found between the mean weighted $\kappa$ value for the 4 point scoring scale ( $\mathrm{KW} 0.49$ ) and the Fisher scoring system ( $\mathrm{KW}$ 0.48). However, a marked improvement in weighted $\kappa$ was found as expected when the more widely varying scores were discussed by the panel of observers and a consensus score agreed ( $\mathrm{Kw} 0.63$ ). Interindividual variation was marked, with an average $\kappa$ statistic of 0.32 (fair), but this may have been exacerbated by the use of two scoring systems.

Variation in weighted $\kappa$ score between the two immunohistochemical methods was noted for the 4 point scoring method, with better scores for EGF receptor staining (mean $\mathrm{kw}$ 0.55 ) than for p53 staining (mean $\mathrm{kw} 0.43$ ). This may reflect difficulties in using the 4 point scoring system for $\mathrm{p} 53$ nuclear staining, which is evaluated above the background colour of the nucleus. Both antibodies produced similar scores when evaluated with the Fisher scoring system. In both scoring systems, better agreement was obtained between observers in scoring the "negative" or " $0+$ " and "weak" or " $1+$ " slides than those which showed stronger staining. This phenomenon of positive staining leading to greater interobserver variation has been noted before. ${ }^{11}$ It is likely to be due to the ease of distinguishing no staining versus the presence of a gradation of colour. It has been suggested as a result that the 4 or 5 point scoring system could be reduced to zero for no staining, 2+ for strong staining, and $1+$ for any staining between these levels. ${ }^{11}$ However, in our study, the three observers found that the Fisher scoring system subjectively provided a better framework for scoring and was much easier to use than a 4 point scale because of the rigidly prescribed definitions provided.

Since immunohistochemical staining techniques are in such widespread use, it is of concern that interobserver error measurements indicate only moderate agreement between observers. In recognition of this, computer assisted image analysis has been employed in some studies. However, it is an expensive and laborious procedure at present and has not yet been validated.

CONCLUSIONS

Until the technology for computer assisted image analysis is validated and becomes widespread, we would recommend the following strategy to ensure reproducible scoring (in conjunction with the EORTC-GCCG task force recommendations):

- Use a panel of tissue control slides to compare with scored slides;

- Use a panel of two or three observers;

- Use highly circumscribed scoring systems, in view of their ease of application;

- Calculate interobserver error, so that the validity of any conclusions is placed in a proper context.

1 Van Diest PJ, van Dam P, Henzen-Logmans SC, et al. A scoring system for immunohistochemical staining: consen-
sus report of the task force for basic research of the EORTC-GCCG. I Clin Pathol 1997;50:801-4.

2 Fisher CJ, Gillett CE, Vojtesek B, et al. Problems with p53 immunohistochemical staining: the effect of fixation and variation in the methods of evaluation. $B r F$ Cancer variation in the

3 Buckley CH, Fox H. Carcinoma of the cervix. In: Anthony PP, Macsween RNM, eds. Recent advances in histopathology, vol 14. London: Churchill, 1989:63-78.

4 Hsu SM, Raine L, Fanger H. A comparative study of the peroxidase-antiperoxidase method and an avidin-biotin complex method for studying polypeptide hormones with radioimmunoassay antibodies. Am f Clin Pathol 1981;75: $734-8$.

5 Altman DG, ed. Practical statistics for medical research, 1st ed. London: Chapman and Hall, 1991.

6 Goppinger A, Wittmaack FM, Wintzer HO, et al. Localisation of human epidermal growth factor receptor in cervical intraepithelial neoplasias. F Cancer Res Clin Oncol 1989; 115:259-63.

7 Holm R, Skomedal H, Helland A, et al. Immunohistochemi$\mathrm{cal}$ analysis of $\mathrm{p} 53$ protein overexpression in normal, premalignant, and malignant tissues of the cervix uteri. $\mathcal{f}$ Pathol 1993;169:21-6.

8 Oka K, Nakano T, Arai T. p53 CM-1 expression is not associated with prognosis in uterine cervical carcinoma. Cancer 1993;72:160-4

9 Kohler M, Janz I, Wintzer HO, et al. The expression of EGF receptors, EGF-like factors and c-myc in ovarian and cervical carcinomas and their potential clinical significance. Anticancer Res 1989;9:1537-48.

10 Landis JR, Koch GG. The measurement of observer agreement for categorical data. Biometrics 1977;33:159-74.

11 van Diest PJ, Weger DR, Lindholm J. Reproducibility of subjective immunoscoring of steroid receptors in breast cancer. Anal Quant Cytol Histol 1996;18:351-4. 